\title{
The Gamma-ray Large Area Space Telescope and Gamma-Ray Bursts
}

\author{
Julie McEnery and Steve Ritz \\ (for the GLAST Mission Team) \\ NASA/GSFC, Lab for Astroparticle Physics, MailCode 661, Greenbelt, MD 20771
}

\begin{abstract}
.
The Gamma-ray Large Area Telescope (GLAST) is a satellite-based observatory to study the high energy gamma-ray sky. The main instrument on GLAST, the Large Area Telescope (LAT) is a pair-conversion telescope that will survey the sky from $20 \mathrm{MeV}$ to greater than $300 \mathrm{GeV}$. With the GLAST launch in 2007, the LAT will open a new and important window on a wide variety of high energy phenomena, including supermassive black holes and active galactic nuclei, gamma-ray bursts, supernova remnants and cosmic ray acceleration and dark matter. A second instrument, the GLAST Burst Monitor (GBM), greatly enhances GLAST's capability to study GRB by providing important spectral and timing information in the $10 \mathrm{keV}$ to $30 \mathrm{MeV}$ range. We describe how the instruments, spacecraft and ground system work together to provide observations of gamma-ray bursts from $8 \mathrm{keV}-300 \mathrm{GeV}$ and to provide rapid notification of bursts to the wider gamma-ray burst community.
\end{abstract}

\section{HIGH ENERGY OBSERVATIONS OF GRB}

EGRET provided a tantalizing glimpse of the properties of gamma-ray bursts above $30 \mathrm{MeV}$. Only a handful were detected, yet the data are consistent with GRB spectra extending with no cutoffs from the sub-MeV range to the high-energy gamma-ray regime. In a couple of GRB, EGRET also discovered bright, high-energy gamma-ray emission with markedly different temporal and spectral characteristics relative to the sub-MeV component. These observations suggest that, at least for some GRB, the bulk of the luminosity is emitted in the $\mathrm{MeV}-\mathrm{GeV}$ range by a separate emission mechanism.

GLAST will address the compelling questions raised by the EGRET observations: How high in energy does the prompt emission extend? Is the hard, independent emission component seen in GRB91017 [1] a common feature of GRB? How common are high energy afterglows such as that seen in GRB940217 [2]?

\section{INSTRUMENTS}

GLAST is a general-purpose gamma-ray facility opening a large new window on the high-energy sky. In this section, we focus only on those aspects of the GLAST instruments that are most relevant to GRB science. 


\section{Large Area Telescope}

Informed by the experience with the highly-successful EGRET instrument on CGRO, the Large Area Telescope (LAT) will provide a large leap in all key capabilities important for burst observations, including

- very large field of view of about $2.5 \mathrm{sr}$, which means that approximately $20 \%$ of all bursts will start within the LAT FOV;

- large effective area, $>8,000 \mathrm{~cm}^{2}$ on-axis for $E$ greater than a few $\mathrm{GeV}$;

- lage acceptance to $E>300 \mathrm{GeV}$, opening the largely-unexplored region above 10 $\mathrm{GeV}$;

- very small deadtime, approximately $26 \mu$ s per event.

Together, these new capabilities will enable the study of high-energy burst emission timing characteristics, and the leap in sensitivity means that more high-energy GRB detections can be anticipated.

More information about LAT performance can be found at http: / / www-glast. slac.stanford.edu/software/IS/glast_lat_performance.htm. The LAT team is a large, international collaboration consisting of more than 120 members from the U.S., Italy, France, Japan, and Sweden. The PI is Peter Michelson (Stanford). The LAT is managed at the Stanford Linear Accelerator Center (SLAC) and is funded by NASA and DOE, along with key international contributions.

\section{GLAST Burst Monitor}

The GBM will provide X-ray and gamma-ray observations of GRB from $<10 \mathrm{keV}$ to $30 \mathrm{MeV}$. It has a very large field of view ( $>8$ steradians) so all GRB within the LAT FOV will have high-energy observations spanning over 7 orders of magnitude in energy. In addition, the GBM will detect and characterize bursts outside the LAT FOV. This information can be used onboard to direct the spacecraft to slew autonomously to bring the GRB within the LAT FOV for bursts deemed to be of high interest. The GBM will detect around 200 GRB per year with $\sim 65$ of these within the canonical LAT FOV. More details about the GBM instrument can be found at http: / / gammaray.msfc . nasa.gov/gbm/.

The development of the GLAST Burst Monitor and analysis of its observational data is a collaborative effort between institutions in the U.S. and Germany. The Principal Investigator is Dr. Charles Meegan at MSFC. Dr. Giselher Lichti at MPE is co-PI.

\section{TYPICAL GLAST GRB TIMELINE}

Upon a burst trigger from either (or both) instrument(s), the observatory will send a sequence of burst alerts to the ground in a similar manner to what is being done for Swift. See Figure 1 for a summary of the processing and messaging timeline. In addition, if the burst is sufficiently interesting, the observatory will repoint autonomously to keep 
Typical GLAST GRB Timeline

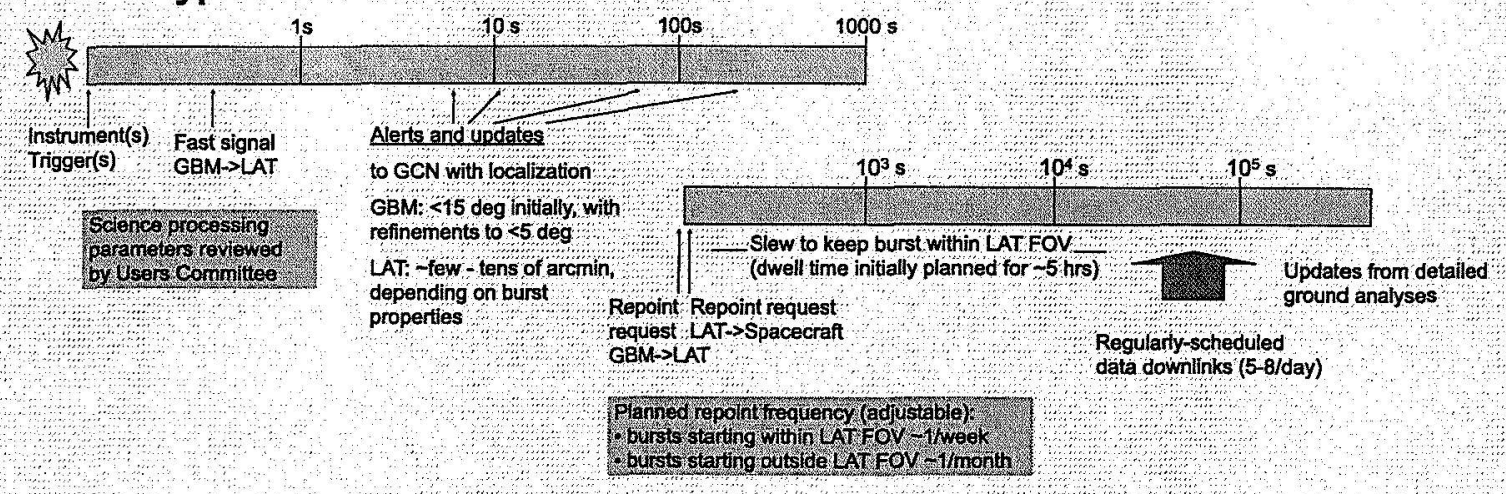

FIGURE 1. GLAST burst processing and action timeline.

the burst within the LAT FOV for a commandable period of time. Based mainly on the remarkable GRB940217[2], from which EGRET observed high-energy emission some 75 minutes after the trigger, the current plan is to set the nominal repoint time to five hours. The autonomous repoint threshold characteristics will be adjusted throughout the mission, in consultation with the community (through the GLAST Users Committee) and the Instrument teams. Bursts that start within the LAT. FOV are intrinsically more interesting; remarkable bursts that start outside the LAT FOV will also trigger a repoint request. The thresholds will therefore initially be adjusted to trigger a repoint for LATFOV bursts approximately once per week and a repoint for a non-LAT-FOV burst once per month.

\section{SCIENCE OPERATIONS}

After inital on-orbit checkout, verification, and calibrations, the first year of science operations will be an all-sky survey. In this mode, the full sky will be covered by the LAT with good uniformity every three hours, with each region of the sky viewed for approximately 30 minutes. The year 1 data will be used for detailed LAT characterization, refinement of the alignment, and key projects including source catalog and diffuse background models, needed by the science community. Thus, during year 1 ,

- summary data on transients, including GRBs, will be released with caveats;

- autonomous repoints for bright bursts will be enabled;

- burst alerts will be enabled;

- extraordinary targets of opportunity will be supported; and

- workshops on science tools, mission characteristics, and tools for proposal preparation will be held for Guest Investigators.

At the end of year 1 , all year 1 data will be released. All subsequent data will be released promptly. The observing plan after year 1 will be driven by Guest Observer proposal selections by peer review, in addition to long periods of sky survey. 


\section{MISSION SCIENCE ELEMENTS}

In addition to the international instrument teams and the GLAST Project Office at Goddard, there are important science elements including:

- The Science Working Group (SWG), whose membership includes the four GLAST Interdisciplinary Scientists, the Users Committee chair, the two Instrument PIs and instrument team representatives, provides advice on mission science requirements and other aspects of the mission implementation.

- The GLAST Users Committee (GUC) provides external review and feedback on the science tools planning and progress. The GUC is also directly involved in community outreach and Guest Investigator opportuntites planning.

- GLAST data and associated analysis software will be provided to the community by the Science Support Center (GSSC), located at Goddard. The GSSC also supports the Guest Investigator program, provides training workshops for the community, and archives the data to the HEASARC.

More information can be found at http://glast.gsfc.nasa.gov and links therein.

\section{SWIFT AND GLAST}

Joint observations of bursts by both Swift and GLAST will be extremely valuable as the two missions provide fundamentally different, but complementary, observations. The two instruments on GLAST and the Swift BAT will provide observations of the prompt phase of GRB over a huge energy range. The GLAST LAT and Swift XRT and UVOT will provide afterglow observations at optical, X-ray and high energy gammaray wavebands. Assuming a Swift detection rate of $100 \mathrm{GRB}$ per year, if the GLAST and Swift pointing directions are uncorrelated, then around 20 Swift GRB per year will occur within the LAT field of view. The fraction of these actually detected by the LAT, and their characteristics, are important questions for GLAST to answer.

\section{REFERENCES}

1. González, M. M., et al, 2003, Nature, 424, 749

2. Hurley, K., et al., 1994, Nature, 372, 652 